\title{
Rompiendo la brecha de la heteronormatividad. Identidad de género y nuevas tendencias sexuales en educación primaria.
}

(Breaking the gap of heteronormativity. Gender identity and new sexual tendencies in primary education.)

Rocío Mercado Colmenero Universidad de Jaén, España.

Fecha recepción: 30-04-2018

Páginas 165-173

Fecha aceptación: 30-06-2018

\section{Resumen.}

Este capítulo pretende mostrar importancia que conlleva en la sociedad eliminar todo tipo de pensamiento heteronormativo en cuanto a identidad y tendencia sexual investigando para ello y desarrollando el concepto de género y la confusión que conlleva ésta con la del sexo, la identidad de género y orientaciones sexuales más allá de las socialmente aceptadas. Tras el estudio de cada tópico se realizará una investigación de tipo cuantitativa a través de una escala liker extraída de los tópicos teóricos explicados anteriormente con cuatro dimensiones, dirigida a alumnos/as del primer curso del Grado en Educación Primaria de la Universidad de Jaén para conocer el nivel de concepción que adquieren sobre esta problemática social, para así poder mostrar conclusiones que serán necesarias para una propuesta de mejora posteriormente en el contexto educativo.

Palabras clave: género; sexo; heteronormatividad; orientación sexual; inclusión educativa

\section{Abstract.}

This chapter aims to show the importance that society has in eliminating all types of heteronormative thinking in terms of identity and sexual orientation by investigating and developing the concept of gender and the confusion that this entails with that of sex, gender identity and sexual orientations beyond the socially accepted. After the study of each topic a quantitative research will be carried out through a liker scale extracted from the theoretical topics explained above with four dimensions, aimed at students of the first year of the Degree in Primary Education of the University of Jaén to know the level of conception that they acquire on this social problem, in order to be able to show conclusions that will be necessary for a proposal of improvement later in the educational context.

Keywords: gender, sex; heteronormativity; sexual orientation; educational inclusion 


\section{1.-Introducción.}

Hasta hace unas décadas la sociedad normalizaba la concepción binaria de género junto con la heterosexualidad. Todo aquello que saliera fuera de lugar de la heteronormatividad conllevaba a una serie de discriminaciones y juicios de valor por parte de la misma. Siguiendo a Millanes (2018) la heteronormatividad es un complejo sistema social, político, económico y cultural regimentado e impuesto en el capitalismo patriarcal, cuyo objetivo es la normalización de las relaciones sexoafectivas y las prácticas sexuales entre personas de distintos sexos.

\section{2.- Adentrándonos en la brecha: Fundamentación Teórica}

\section{1.-Concepción de género.}

Según Lamas (2013) el género se contextualiza como el cúmulo de ideas, formas, destrezas y mandatos sociales que una cultura desenvuelve desde la diferencia anatómica entre mujeres y hombres, para figurar y construir socialmente lo que es propio de los hombres (lo masculino) y propio de las mujeres (lo femenino), tal como defiende De la Torre (2017) enfocando al género como un proceso de desigualdad en la sociedad, adentrándonos en el concepto de identidad de género ya que en el momento de nacer biológicamente nacemos hombre y mujer, asociando lo femenino a la mujer y lo masculino al hombre, por lo que la sociedad ha asignado a lo biológico lo que le corresponde como atributo social.

El género es un término que ocasiona complicaciones en cuanto a su entendimiento siendo éste un concepto que etiqueta a un individuo en cuanto a que tipo o clase de especie pertenece. Como cúmulo de personas con un sexo común se trata de las mujeres y hombres, como género nos referimos a género femenino y género masculino. (Lamas, 2017)

\section{2.-Teoría sexo y Género}

Rosales y Salinas (2017) definen al sexo como al cúmulo de particularidades físicas, biológicas, anatómicas y fisiológicas del ser humano, siendo éstas las que asignan al sujeto como hombre o como mujer. El sexo viene establecido por la naturaleza siendo una condición biológica con la que nacemos. Aunque se nazca con el sexo asignado es posible modificarlo ya que la propia identidad de género es un derecho del ser humano y mediante un proceso quirúrgico.

Continuando con Martell, Ibarra, Conteras y Camacho (2018) podemos destacar que existen tres categorías en cuanto a la propia identificación con el propio sexo:

- Sexo Biológico: Refiriendo a la condición física y corpórea de una persona.

Se trata de características biológicas que demuestran diferencias entre hombre y mujeres más allá de los órganos sexuales como el sexo gonadal, el sexo cromosómico (en la mujer XY y en los hombres XX) y el sexo fenotípico (Disposición de los órganos reproductores internos y externos). 
- Sexo Psicológico: Es el cúmulo de las experiencias y vivencias psíquicas de una persona como mujer y como hombre. Es el proceso en el que el/la sujeto toma conciencia de pertenecer a un sexo específico donde puede coincidir o no con el sexo biológico, esta toma de conciencia tiene a estar influenciada por el contexto familiar, social y educativo.

- Sexo Sociológico: Es el sexo que se le atribuye al sujeto en el momento de su nacimiento. Es la manera en la que las personas de su alrededor identifican el/la sujeto. Se tiende a especificar los estereotipos que se le designan a las personas dependiendo de la sociedad en la que conviven y/o van a convivir para fortalecer en cierta manera la propia identidad desde la infancia hasta la adolescencia (concienciación de "uno mismo").

\section{3.- Sistema de género binario.}

La sociedad occidental presenta un sistema binario debido a la naturalización de identidades a través del sexo biológico porque se entiende que el sexo se relaciona con los aparatos reproductores y como tales, solo existen dos, por lo que se defiende la idea de la existencia de dos sexos. En el caso hipotético de que existieran más se amenazaría la procreación, aspecto importante y con lo cual se justifica en muchas ocasiones la contra sobre la diversidad sexual (Calvo 2018)

La efectividad de este sistema binario se debe a las destrezas de tipo social que interioriza el/la sujeto, dichas destrezas nos instruyen a ser mujeres y a ser hombres. Esta idea es equívoca porque el desarrollo de la identidad se trata de un proceso individual basado en la tolerancia y respeto de que cada uno/a elige lo que más le satisfaga. La asignación del sistema de género binario está tan enraizada y habituada que se aprende de manera normalizada, cuando ésta provoca discriminaciones a la hora de categorizar y etiquetar cualquier identidad de género que se considere "fuera de lo común". Este hecho lo provoca una visión heterogénea capaz de rechazar cualquier posición y reflexión alejada de la asignación binaria de género. (Calvo 2018).

\section{4.- Identidad de género.}

La actual sociedad está tomando conciencia del proceso que conlleva adquirir la propia identidad independientemente del sexo adquirido al nacer. Este proceso conlleva a perder el miedo y la vergüenza debido al deseo de libertad de expresión que interioriza el ser humano (Granados, Hernández y Olvera 2017). Existe un gran porcentaje de población que no está conforme con su género al pertenecer al sexo opuesto. Siguiendo a Granados, Hernández y Olvera (2017) podemos detallar los siguientes conceptos:

- Cisgénero: es aquella persona que se siente cómoda y conforme con su sexo y acepta los roles establecidos en cuanto al género correlacionado.

- Transexual: deseo y convicción de pertenecer al sexo opuesto. Se trata de toda persona que no se siente clarificado con el sexo que ha nacido ni con 
su cuerpo, por lo que requiere de una transformación de cuerpo y de identidad. Estas personas cambian aparte de sus genitales, el estilo de vida, comportamiento, estética y su desarrollo social. Este proceso de cambio se denomina proceso transexualizadorbasándose en una terapia hormonal que deriva a una operación de cambio de sexo. Cuando este cambio se produce en la edad adulta se le conoce como transexualismo secundario.

- Transgénero: se refiere a todo colectivo de personas que se sienten identificadas con el sexo opuesto y desean pertenecer a él sin necesidad de reasignación de sexo, es decir, sin proceso hormonal que derive a operación. En cuanto a orientación sexual no es relevante si nos referimos a identidad.

- $\quad$ Travesti: es aquella identidad de género que se base en el comportamiento ya sea hombre o mujer. Es aquella muestra de identidad a partir de la forma de vestir, roles de comportamiento y actitud. No siempre tiene por qué derivar al deseo de pertenecer o identificarse con el sexo opuesto.

\section{5.-Orientación sexual y tipos: fragmentando la heteronormatividad.}

La orientación sexual según muestra Pérez (2014) es la atracción hacia otra persona de tipo emocional, sexual, afectiva, personal y romántica debido a factores biológicos, psicológicos, económicos, culturales, religiosos y sociales. Antiguamente solo era aceptada si se trataba de heterosexualidad. Aún así, es de cierta complejidad poder categorizar cada una de las orientaciones sexuales ya que se conocen actualmente trece tipos (contando con la heterosexualidad, bisexualidad y homosexualidad.

Colectivos sociales como es el LGTBIQ, han sido una pieza fundamental en la sociedad para clarificar muchos de los conceptos actuales y para promover la libertad y derechos que tenemos los ciudadanos. Como ya se ha mencionado, aunque solo se conozca la división entre homosexualidad y heterosexualidad, progresivamente han surgido más tendencias sexuales (Pérez 2014).

- Antrosexualidad: una persona antrosexual es aquella persona que desconoce aún su tendencia sexual por otras personas pero son flexibles a la hora de crear vínculos con otras personas.

- Asexualidad: se considera como la falta de orientación sexual, las personas asexuales no sienten ninguna atracción física, social ni sexual por ningún sujeto.

- $\quad$ Autosexualidad: es aquella atracción que padece el sujeto por sí mismo. El autoerotismo es un factor imprescindible en esta tendencia sexual.

- Demisexualidad: la demisexualidad expone que es una atracción por personas que conlleva un vínculo romántico, íntimo y emocional, es decir, solo existe atracción una vez que se haya creado un vínculo emocional de confianza.

- Graysexualidad: es aquella tendencia sexual que se situa entre la asexualidad y la sexualidad, va por etapas. el sujeto no tiene por qué siempre situarse en una sexualidad continua lo mismo que puede elegir por 
sí mismo en un periodo de su vida no mantener ninguna atracción física, sexual ni social.

- Hiposexualidad: las personas hiposexuales son aquellas que tienen poco apetito sexual con otras personas y el autoerotismo también es escaso. Se confunde mucho este término con la asexualidad, lo que diferencia la hiposexualidad de ésta es que se debe a los genes, exceso de trabajo y estrés.

- Lithsexualidad: aquellas personas lithsexuales son las que sienten una atracción por otra persona sin tener la necesidad de ser correspondidas.

- Pansexualidad: esta orientación genera mucha confusión con la bisexualidad, una persona pansexual siente una atracción sexual y sentimental hacia otras personas sin importar su sexo y género.

- Sapiosexualidad: los sapiosexuales se conocen como aquellas personas que se sienten atraídos por la inteligencia cognitiva y emocional de una persona, es vínculo exclusivamente intelectual.

- Skoliosexualidad: es la tendencia sexual hacia personas transgénero, transexuales e intergénero (identidad de género que se sitúa entre los dos géneros), es decir, es la atracción sexual hacia personas no cisgénero.

\section{3.-Metodologia.}

Esta investigación es de naturaleza exploratoria y descriptiva, se adoptará una metodología cuantitativa, pues lo que queremos es mostrar la formación que sobre identidad de género tienen los futuros docentes, determinar las variadas orientaciones sexuales en nuestro contexto social, así como las variantes de tendencia sexual que podemos encontrar en la sociedad actual.Para la realización de la investigación se opta por la utilización de una escala como instrumento de recogida de datos.

\section{4.- Problema de investigación}

¿Los futuros docentes de educación primaria tienen conocimientos básicos sobre libertad, identidad y tendencia sexuales para dar respuesta a las necesidades de sus alumnos y alumnas?

\section{5.-Análisis de datos.}

Vamos a realizar el análisis de los datos ofrecidos por la escala Likert, en función de cada objetivo especifico

- O.E.1.-Revisar los conocimientos que los futuros docentes de educación primaria tienen sobre sexo y género. 


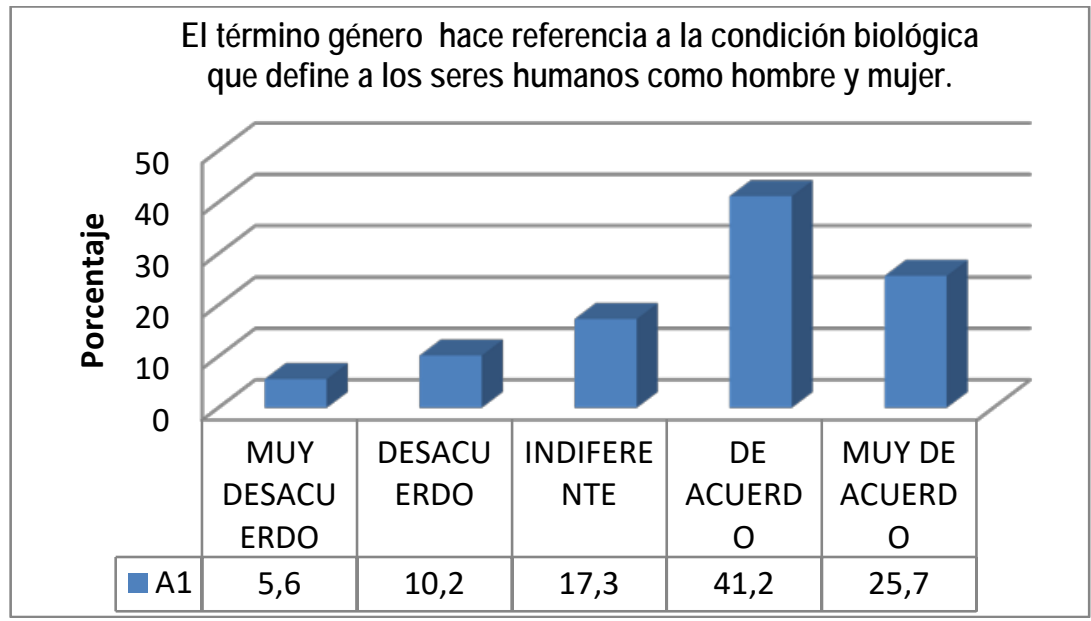

Fuente: La propia encuesta (2018).

Un $41.2 \%$ responde de acuerdo a que el término género hace referencia a la condición biológica que define a los seres humanos como hombre y mujer, podemos concluir que los encuestados confunden los términos sexo y género, en realidad es el sexo el término que hace referencia a la condición biológica que define a los seres humanos como hombre y mujer

- O.E.2.-Mostrar la formación que sobre identidad de género tienen los futuros docentes.

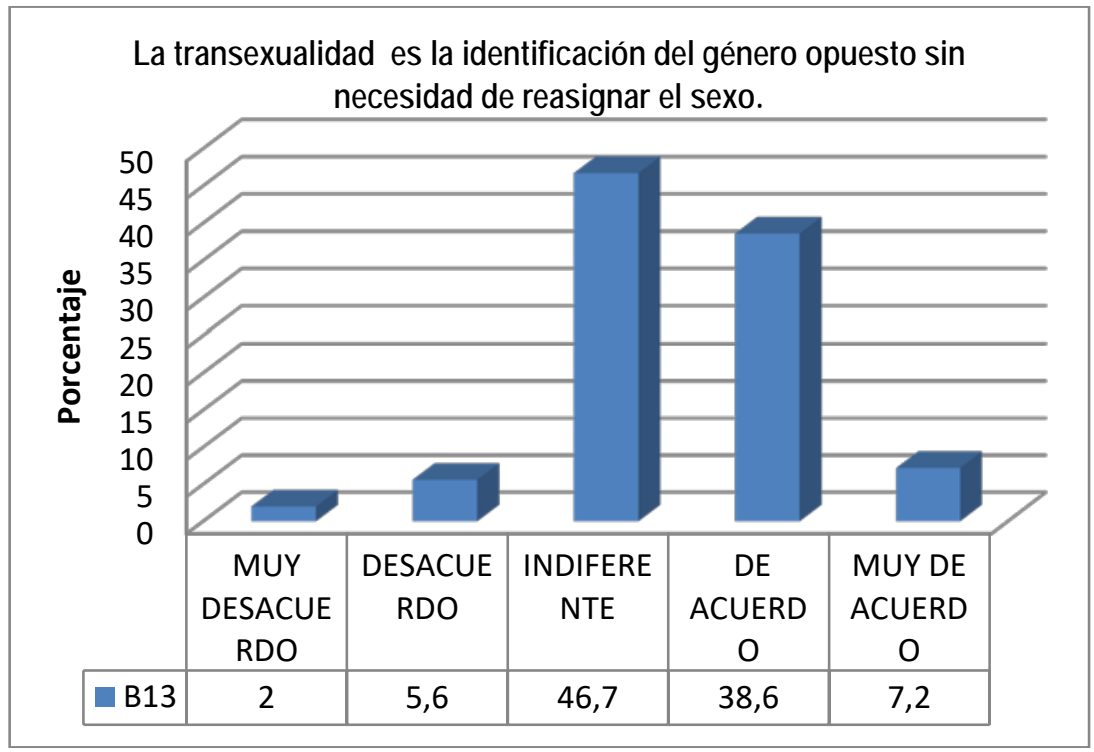


Fuente: La propia encuesta (2018).

Si analizamos bien, los mayores porcentajes corresponden a las respuestas indiferente $(46,7 \%)$ y de acuerdo $(38,6)$ cuando se trata en realidad de personas transgénero ya que son aquellas que se identifican con el sexo opuesto sin necesidad de llevar a cabo un proceso de reasignación de sexo.

- O.E.3.-Determinar las variadas orientaciones sexuales en nuestro contexto social.

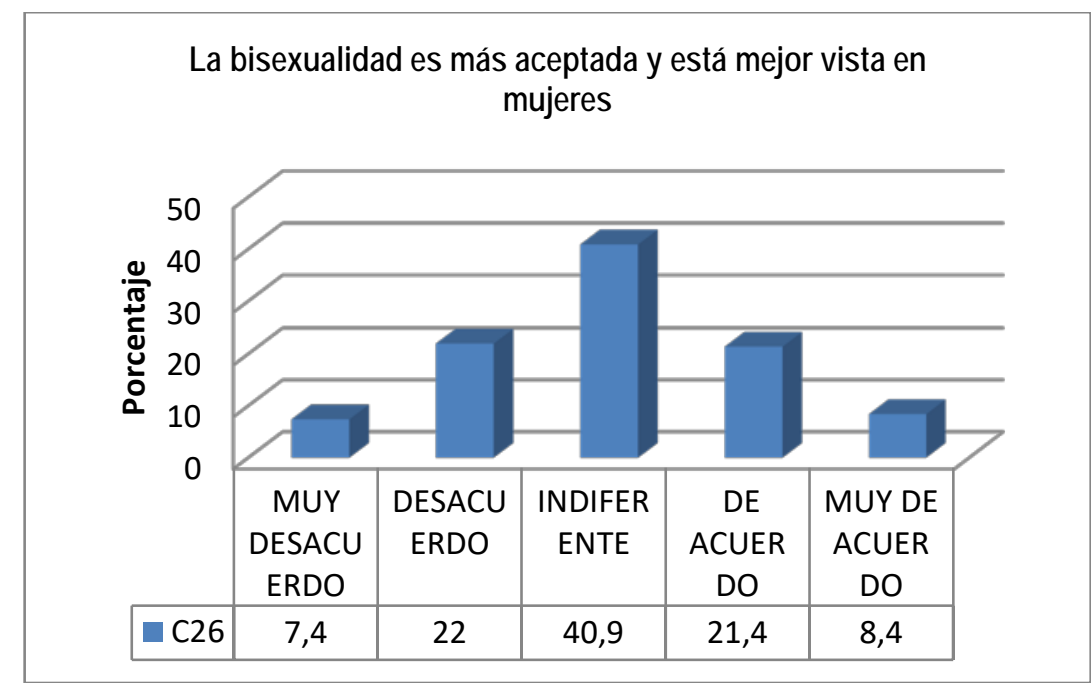

Fuente: La propia encuesta (2018).

Tan solo un 7.4\% es capaz de ver que esta expresión tiene de base la ideología de la heteronormatividad aparte de ser un falso mito ya que la bisexualidad tiene ser aceptada de la misma manera en hombres y mujeres.

- O.E.4.-Determinar las variantes de tendencia sexual que podemos encontrar en la sociedad actual. 


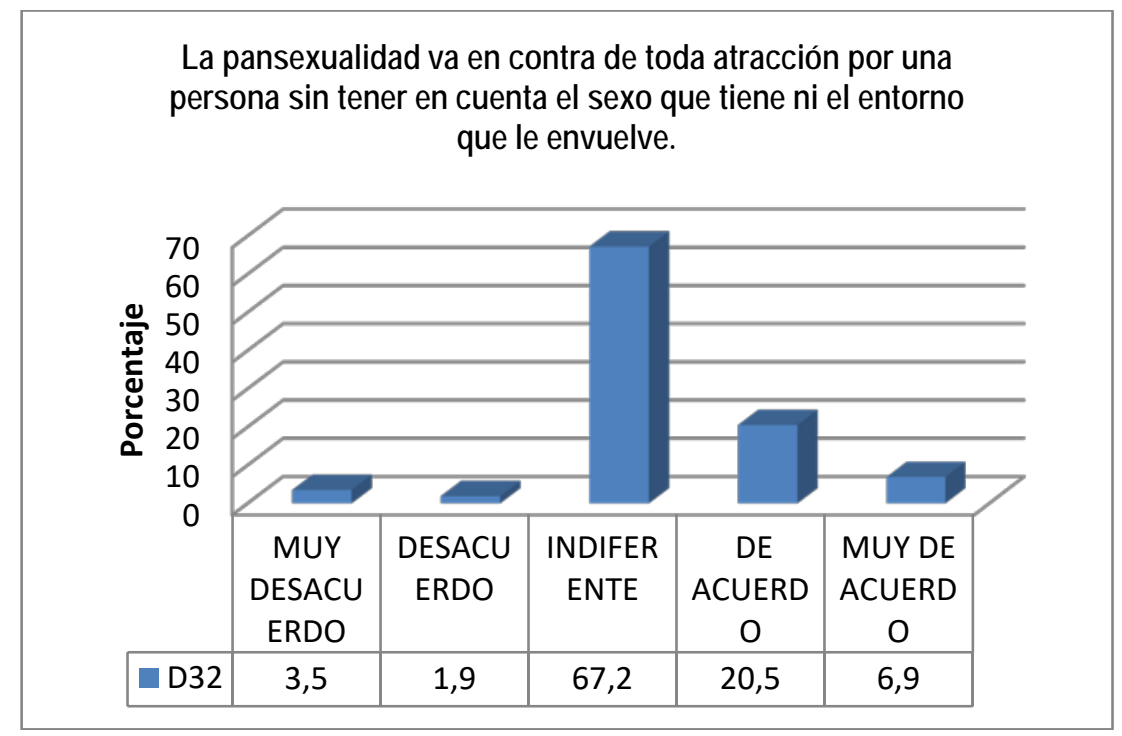

Fuente: La propia encuesta (2018).

Si observamos cada una de las respuestas, podemos destacar que no hay conciencia ninguna que sobresalga de las orientaciones heteronormativas establecidas, todo aquello que sobresalga de la homosexualidad y heterosexualidad supone un escaso conocimiento sobre ello. Tan solo un 6.9\% es capaz de contestar a la verdadera respuesta sobre la pansexualidad

\section{6.-Conclusión.}

Aunque se está viviendo un cambio radical en cuanto a la libertad de identidad y tendencia sexuales y existan numerosas campañas y aportaciones para facilitar la total comprensión de éstas puedo exponer con certeza que queda mucho camino que recorrer. Se requiere una total comprensión, tolerancia y respeto en cuanto a la identidad de cada uno/a, es de real importancia interiorizar que no solo existe un camino en cuanto a la orientación y atracción sexual, abarcando más factores como el emocional y el psicológico. Es imprescindible mostrar estas ideas y concepciones en ámbitos de nuestro día a día como es el contexto familiar y educativo partiendo siempre de valores como la empatía y tolerancia para así poder educar a las personas para su desarrollo integral en la sociedad sin discriminaciones y juicios de valor. La educación es la herramienta más valiosa y los/las docentes los mejores guías para ayudar a este progreso, de ahí resaltar e investigar sobre el nivel de concepción de los/las futuros/as docentes para mejorar la formación de éstos/éstas ante estos conceptos, siendo necesario que los/las alumnos/as puedan reflexionar críticamente sobre estos conceptos y que puedan entender que pueden ser lo que ellos quieran rompiendo las brechas creadas por la sociedad que solamente impulsan la heteronormatividad. 


\section{7.-Referencias}

Calvo (2018) Las identidades de género según las y los adolescentes. Percepciones, desigualdades y necesidades educativas.Contextos Educativos (21) 169-184

De la Torre, V.M. (2017). Identidad de género, una categoría para la deconstrucción. Revista Xihmai XII (23), 83-102.

Granados, J.A., Hernández Ramírez P.A., Olveda O.A. (2017). Perfomatividad del género, medicalización y salud en mujeres transexuales en Ciudad de México. Salud Colectiva (4) 633-646

Lamas, M. (2017). El género. La construcción cultural de la diferencia sexual. México: Bonilla Artigas.

Lamas, M. (2013). La construcción cultural de la diferencia sexual. México: Porrúa.

Martell, N.G., Ibarra, M.L., Comtreras, G., Camacho, E.J. (2018). La sexualidad en adolescentes desde la teoría de las representaciones sociales. Psicología y salud. (28) 15-24

Pérez Borge, D. (2014). La orientación sexual como continuo. Revista insight. España

Rosales Mendoza, A.L., Salinas Quiroz, F. (2017) Educación sexual y género en primarias mexicanas ¿qué dicen los libros de texto y el profesorado? Educare Electronic Journal (21), 1-21 